\title{
Universal shocks in random matrix theory
}

\author{
Jean-Paul Blaizot ${ }^{1,}$, and Maciej A. Nowak ${ }^{2, \text {, }}$ \\ ${ }^{1}$ IPTh, CEA-Saclay, 91191 Fif-sur Yvette, France \\ ${ }^{2}$ M. Smoluchowski Institute of Physics and Mark Kac Center for Complex \\ Systems Research, Jagiellonian University, PL-30-059 Cracow, Poland
}

(Dated: June 4, 2018)

\begin{abstract}
We link the appearance of universal kernels in random matrix ensembles to the phenomenon of shock formation in some fluid dynamical equations. Such equations are derived from Dyson's random walks after a proper rescaling of the time. In the case of the Gaussian Unitary Ensemble, on which we focus in this letter, we show that the orthogonal polynomials, and their Cauchy transforms, evolve according to a viscid Burgers equation with an effective "spectral viscosity" $v_{s}=1 / 2 N$, where $N$ is the size of the matrices. We relate the edge of the spectrum of eigenvalues to the shock that naturally appears in the Burgers equation for appropriate initial conditions, thereby obtaining a new perspective on universality.
\end{abstract}

PACS numbers:

In a seminal paper [1], Dyson showed that the distribution of eigenvalues of a random matrix could be interpreted as the result of a random walk performed independently by each of the matrix elements. The resulting distribution yields the so called "Coulomb gas" picture, with the eigenvalues identified to charged point particles repelling each other according to Coulomb law. For matrices of large sizes, this correctly describes the bulk properties of the spectrum [2]. In his original work Dyson introduced a restoring force preventing the eigenvalues to spread for ever as time goes. This is what allowed him to find a stationary solution corresponding to the random ensemble considered with a chosen variance (related to the restoring force). In this note, we point out that a richer structure emerges if one ignores the restoring force and performs a rescaling of the time of the random walk. The random walk can then be described by an equation of fluid dynamics, the viscid Burgers equation. In this picture, that we may refer to as "Dyson fluid", the edge of the spectrum appears as the precursor of a shock wave, and its universal properties follow from a simple analysis of the Burgers equation that was developed in other contexts [3]. This enables us to recover familiar results of random matrix theory in a simple way, and get a new perspective on the issue of universality.

In this letter we shall consider only the Gaussian Unitary Ensemble, although we believe that many of our results can be extended to other ensembles. Thus, we consider $N \times N$ hermitian matrices $H$ with complex entries. We assume that these matrices evolve in time according to the following random walk: in the time step $\Delta \tau$ the matrix elements change according to $H_{i j} \rightarrow H_{i j}+\delta H_{i j}$, with $\left\langle\delta H_{i j}\right\rangle=0$, and $\left\langle\left(\delta H_{i j}\right)^{2}\right\rangle=$ $\left(1+\delta_{i j}\right) \Delta t$. That is, we assume that at each time step, the increment of the matrix elements follows from a Gaussian distribution with a variance proportional to $\Delta t$. The initial condition on the random walk is that at time $t=0$, all the matrix elements vanish. Alternatively, let $x_{i}$ denote the eigenvalues of $H$. The previous random walk translates into a corresponding

*Electronic address: Jean-Paul.Blaizot@cea.fr

${ }^{\dagger}$ Electronic address: nowak@th.if.uj.edu.pl random walk of the eigenvalues, with the following characteristics [1]

$$
\left\langle\delta x_{i}\right\rangle=E\left(x_{i}\right) \Delta t, \quad\left\langle\left(\delta x_{i}\right)^{2}\right\rangle=\Delta t,
$$

where the "Coulomb force"

$$
E\left(x_{j}\right)=\sum_{i \neq j}\left(\frac{1}{x_{j}-x_{i}}\right)
$$

originates in the Jacobian of the transformation from the matrix elements to the eigenvalues, $\Delta=\prod_{i<j}\left(x_{i}-x_{j}\right)^{2}$. The probability for finding the set of eigenvalues $x_{1}, \cdots, x_{N}$ at time $t$ obeys the Smoluchowski-Fokker-Planck equation

$$
\frac{\partial P}{\partial t}=\frac{1}{2} \sum_{i} \frac{\partial^{2} P}{\partial x_{i}^{2}}-\sum_{i} \frac{\partial}{\partial x_{i}}\left(E\left(x_{i}\right) P\right),
$$

whose solution reads

$$
P\left(x_{1}, \cdots, x_{N}, t\right)=C \prod_{i<j}\left(x_{i}-x_{j}\right)^{2} \mathrm{e}^{-\sum_{i} \frac{x_{i}^{2}}{2 t}},
$$

with $C$ a (time-dependent) normalization constant.

The average density of eigenvalues, $\tilde{\rho}(x)$, may be obtained from $P$ by integrating over $N-1$ variables. Specifically:

$$
\tilde{\rho}(x, t)=\int \prod_{k=1}^{N} d x_{k} P\left(x_{1}, \cdots, x_{N}, t\right) \sum_{l=1}^{N} \delta\left(x-x_{l}\right),
$$

with normalization $\int d x \tilde{\rho}(x)=N$. Similarly we define the "two-particle" density $\tilde{\rho}(x, y)=\left\langle\sum_{l=1}^{N} \sum_{j \neq l} \delta\left(x-x_{l}\right) \delta\left(y-x_{i}\right)\right\rangle$, with $\int d x d y \tilde{\rho}(x, y)=N(N-1)$. These various densities obey an infinite hierarchy of equations obtained form Eq. (3) for $P$. Thus, the equation relating the one and two particle densities reads

$$
\frac{\partial \tilde{\rho}(x, t)}{\partial t}=\frac{1}{2} \frac{\partial^{2} \tilde{\rho}(x, t)}{\partial \lambda^{2}}-\frac{\partial}{\partial \lambda} f_{d y} \frac{\tilde{\rho}(x, y, t)}{x-y},
$$

where $f$ denotes the principal value of the integral. 
In the large $N$ limit, this equation becomes a closed equation for the one particle density. To show that, we set

$$
\tilde{\rho}(x, y)=\tilde{\rho}(x) \tilde{\rho}(y)+\tilde{\rho}_{c o n}(x, y),
$$

where $\tilde{\rho}_{c o n}(x, y)$ is the connected part of the two-point density. Then we change the normalization of the single particle density, defining

$$
\tilde{\rho}(x)=N \rho(x),
$$

and similarly $\tilde{\rho}(x, y)=N(N-1) \rho(x, y)$. At the same time, we rescale the time so that $\tau=N t[7]$. One then obtains

$$
\frac{\partial \rho(x)}{\partial \tau}+\frac{\partial}{\partial x} \rho(x) f d y \frac{\rho(y)}{x-y}=\frac{1}{2 N} \frac{\partial^{2} \rho(x)}{\partial x^{2}}+f d y \frac{\rho_{c o n}(x, y)}{x-y} \text {. (9) }
$$

In the large $N$ limit, the right hand side is negligible, leaving as announced a closed equation for $\rho(x, \tau)$. This equation can be further transformed into an equation for the resolvent

$$
G(z, \tau)=\left\langle\frac{1}{N} \operatorname{Tr} \frac{1}{z-H(\tau)}\right\rangle=\int d y \frac{\rho(y, \tau)}{z-y},
$$

whose imaginary part for $z=x-i \epsilon$, and $x$ real, yields the average spectral density $\rho(x)$, and the real part is the Hilbert transform of $\rho, \mathcal{H} \rho(x)=f d y \rho(y) /(x-y)$. By taking the Hilbert transform of Eq. (9), keeping only the dominant term in the large $N$ limit and using well known properties of the Hilbert transform, one gets the following equation for $G(z, \tau)$

$$
\partial_{\tau} G(z, \tau)+G(z, \tau) \partial_{z} G(z, \tau)=0 .
$$

This is the inviscid Burgers equation [6]. Note that the Laplacian, that naturally appears in the description of diffusive processes, has disappeared in the large $N$ limit, as already indicated after Eq. (9). The non-linear term plays the role of an effective mean field representing the mutual repulsion of the evolving eigenvalues. We shall return later to the role of diffusion, and focus now on the solution of Eq. (111).

This can be obtained by using the method of (complex) characteristics [9], with the characteristics determined by the implicit equation $z=\xi+\tau G_{0}(\xi)$, where $G_{0}(z)=G(z, \tau=$ $0)=1 / z$. Assuming the solution $\xi(z, \tau)$ to be known, the Burgers equation can be solved parameterically as $G(z, \tau)=$ $G_{0}(\xi(z, \tau))=G_{0}(z-\tau G(z, \tau))$. The solution of this equation that is analytic in the lower half plane is

$$
G(z, \tau)=\frac{1}{2 \tau}\left(z-\sqrt{z^{2}-4 \tau}\right),
$$

whose imaginary part yields the familiar Wigner's semicircle for the average density of eigenvalues. In the fluid dynamical picture suggested by the Burgers equation, the edge of the spectrum corresponds to a singularity that is associated with the precursor of a shock wave, sometimes referred to as a "pre-shock" [3]. This singularity occurs when the mapping between $z$ and $\xi$ ceases to be one-to-one, a condition required for the validity of the method of characteristics. This takes place when $d z / d \xi=0=1+\tau G_{0}^{\prime}\left(\xi_{c}\right)$, defining $\xi_{c}(\tau)$. Since $G_{0}^{\prime}\left(\xi_{c}\right)=-1 / \xi_{c}^{2}, \xi_{c}(\tau)= \pm \sqrt{\tau}$, and $z_{c}=\xi_{c}+\tau G_{0}\left(\xi_{c}\right)= \pm 2 \sqrt{\tau}$.
That is, the singularity occurs precisely at the edge of the spectrum. Furthermore, the resulting singularity is of the square root type. To see that, let us expand the characteristic equation around the singular point. We get

$$
z-z_{c}=\frac{\tau}{2}\left(\xi-\xi_{c}\right)^{2} G_{0}^{\prime \prime}\left(\xi_{c}\right)=\frac{\tau}{\xi_{c}^{3}}\left(\xi-\xi_{c}\right)^{2} .
$$

It follows that, in the vicinity of the positive edge of the spectrum $z \simeq z_{c}=2 \sqrt{\tau}, \xi-\xi_{c}= \pm \tau^{1 / 4} \sqrt{z-z_{c}}$. Thus, as $z$ moves towards $z_{c}$ and is bigger than $z_{c}, \xi$ moves to $\xi_{c}$ on the real axis. When $z$ becomes smaller than $z_{c}, \xi$ moves away from $\xi_{c}$ along the imaginary axis. The imaginary part therefore exists for $z<z_{c}$ and yields a spectral density $\rho(z) \sim \sqrt{z_{c}-z}$, in agreement with (12). This square root behaviour of the spectral density implies that in the vicinity of the edge of the spectrum, the number of eigenvalues in an interval of width $s$ scales as $N s^{3 / 2}$, implying that the interlevel spacing goes as $N^{-2 / 3}$.

In order to capture the fine structure of the level density in the vicinity of the edge, we need to take into account the $1 / N$ corrections. We wish to do that within the Dyson fluid picture. Trying to go beyond the large $N$ limit at the level of Eq. (9) requires in particular handling the connected two-point function. We shall not do so directly, but shall rely on time dependent orthogonal polynomials, i.e., extend to our timedependent setting tools that are familiar in random matrix theory. This will allow us to obtain simple equations that generalize Eq. (9) (albeit not for the one-particle density), take the form of viscid Burgers equations, and are valid for any $N$. This procedure allows, in principle, the calculation of all the correlation functions for finite $N$ and $t$.

For the considered ensemble, the relevant set of orthogonal polynomials is that of Hermite polynomials, defined as [4]

$$
h_{k}(x)=(-1)^{k} e^{N x^{2} / 2} \frac{d^{k}}{d x^{k}} e^{-N x^{2} / 2} .
$$

These admit the following useful integral representation

$$
h_{k}(x)=(-i N)^{k} \sqrt{\frac{N}{2 \pi}} e^{N x^{2} / 2} \int_{-\infty}^{\infty} d q q^{k} e^{-N / 2 q^{2}+i x q N} .
$$

We shall also use the so-called monic polynomials, i.e. polynomials where the coefficient of highest order term is equal to unity: $\pi_{k}(x) \equiv h_{k}(x) / N^{k}=\prod_{i=1}^{k}\left(x-\bar{x}_{i}\right)$, with $\bar{x}_{i}$ denoting the (real) zeros of the Hermite polynomials. In the random walk described above, the probability distribution retains its form at all instants of time. It is then easy to construct polynomials that remain orthogonal with respect to the time-dependent measure $\exp \left(-N x^{2} /(2 \tau)\right)$ : all that needs to be done is to replace $N$ by $N / \tau$ in Eq. (15). One obtains then

$$
\pi_{k}(x, \tau)=\frac{(-i)^{k}}{k !} \sqrt{\frac{N}{2 \pi \tau}} \int d q q^{k} e^{-\frac{N}{2 \tau}(q-i x)^{2}},
$$

which satisfy

$$
\int_{-\infty}^{\infty} d x \mathrm{e}^{-\frac{N x^{2}}{2 \tau}} \pi_{n}(x, \tau) \pi_{m}(x, \tau)=\delta_{n m} c_{n}^{2}
$$


with $c_{n}^{2}=n ! \sqrt{2 \pi} \tau^{n+1 / 2}$. The monic character of the $\pi_{n}$ 's is not affected by the time dependence. By using the integral representation (16), it is easy to show that the $\pi_{n}(x, \tau)$ 's satisfy the following equation

$$
\partial_{\tau} \pi_{n}(x, \tau)=-v_{s} \partial_{x}^{2} \pi_{n}(x, \tau),
$$

with $v_{s}=1 / 2 N$. This is like a diffusion equation with however a negative diffusion constant (note however that $\pi_{n}$ is an analytic function of $x$, and that $\pi_{n}(-i y, \tau)$, with $y$ real, satisfies a diffusion equation with a positive constant). At this point, one may perform an inverse Cole-Hopf transform, i.e. , define the new function

$$
f_{k}(z, \tau) \equiv 2 v_{s} \partial_{z} \ln \pi_{k}(z, \tau)=\frac{1}{N} \sum_{i=1}^{k} \frac{1}{z-\bar{x}_{i}(\tau)},
$$

with $\operatorname{Im} z \neq 0$. The resulting equation for $f_{k}$ is the viscid Burgers [5] equation

$$
\partial_{\tau} f_{k}(z, \tau)+f_{k}(z, \tau) \partial_{z} f_{k}(z, \tau)=-v_{s} \partial_{z}^{2} f_{k}(z, \tau),
$$

with $v_{s}$ playing the role of a viscosity.

The equation (20) is satisfied by all the functions $f_{k}$. We shall focus now on the function $f_{N}$ associated to $\pi_{N}(z, \tau)$. One reason is that $\pi_{N}(z, \tau)$ is known to be equal to the average characteristic polynomial [8], i.e

$$
\langle\operatorname{det}(z-H(\tau))\rangle=\pi_{N}(z, \tau),
$$

and, in the large $N$ limit, $(1 / N) \partial_{z} \ln \langle\operatorname{det}(z-H(\tau))\rangle \approx$ $(1 / N) \partial_{z}\langle\ln \operatorname{det}(z-H(\tau))\rangle=G(z)$. Thus $f_{N}(z, \tau)$ coincides with the average resolvent $G(z, \tau)$ in the large $N$ limit. In fact the structure of $f_{N}$, as clear from Eq. (19), is very close to that of the resolvent, with its poles given by the zeros of the characteristic polynomial. Eq. (20) for $f_{N}(z, \tau)$ is exact. The initial condition, $f_{N}(z, \tau=0)=1 / z$, does not depend on $N$, so that all the finite $N$ corrections are taken into account by the viscous term. Note however that this exact equation does not allow us to directly study the finite $N$ corrections to the resolvent or the spectral density.

We turn now to the study of the viscid Burgers equation for $f_{N}(z, t)$, i.e. Eq, (20) for $k=N$, in the vicinity of the (moving) pre-shock, that is for $x$ near $z_{c}(\tau)$. The Cole-Hopf transformation used above provides a solution in term of $\pi_{N}(x, \tau)$ which allows us to study the effects of a small viscosity by performing a saddle point approximation on the integral (16). The saddle point equation coincides with the characteristic equation discussed earlier (with the identification $\xi \rightarrow-i q$ ). However this analysis breaks down in the vicinity of the shock: here the difficulties comes from the merging of the two saddle points associated with the square root singularity. A better analysis is then called for, and we shall rely in what follows on tools borrowed from the theory of turbulence [10]. Let us recall that in the vicinity of the edge of the spectrum, and in the inviscid limit,

$$
f_{N}(z, \tau) \simeq \pm \frac{1}{\sqrt{\tau}} \mp \frac{1}{\tau^{3 / 4}} \sqrt{z-z_{c}} .
$$

We set

$$
x=z_{c}(\tau)+v_{s}^{2 / 3} s, \quad f_{N}(x, \tau)=\dot{z}_{c}(\tau)+v_{s}^{1 / 3} \chi_{N}(s, \tau),
$$

with $\dot{z}_{c} \equiv \partial_{\tau} z_{c}= \pm 1 / \sqrt{\tau}$. The particular scaling of the coordinate is motivated by the fact that near the square root singularity the spacing between the eigenvalues scales as $N^{-2 / 3}$. A simple calculation then yields the following equation for $\chi(s, \tau)$ in the vicinity of $z_{c}(\tau)=2 \sqrt{\tau}$ :

$$
\partial_{\tau}^{2} z_{c}+v_{s}^{1 / 3} \frac{\partial \chi}{\partial \tau}+\chi \frac{\partial \chi}{\partial s}=-\frac{\partial^{2} \chi}{\partial s^{2}},
$$

which, ignoring the small term of order $v_{s}^{1 / 3}$, we can write as

$$
\frac{\partial}{\partial s}\left[-\frac{s}{2 \tau^{3 / 2}}+\frac{1}{2} \chi^{2}+\frac{\partial \chi}{\partial s}\right]=0,
$$

or, setting $\chi=2 \partial_{s} \ln \phi(s)$, as

$$
\partial_{s}^{2} \phi-a\left(s-s_{0}\right) \phi=0, \quad a \equiv \frac{1}{4 \tau^{3 / 2}},
$$

with $s_{0}(\tau)$ an arbitrary function of $\tau$. The general solution of this equation is given by Airy functions [27] $\phi_{i}(s)=$ $\epsilon_{i} A i\left(\epsilon_{i}\left(s-s_{0}\right) a^{1 / 3}\right)$, where $\epsilon_{i}$ for $i=0,1,2$ are cubic roots of 1 and $\sum_{0}^{2} \phi_{i}(s)=0$. In the case of the characteristic polynomial, the solution corresponds to $\epsilon_{0}=1$. Finally,

$$
\chi(s, \tau)=2 \frac{A i^{\prime}\left(a^{1 / 3}\left(s-s_{0}\right)\right)}{A i\left(a^{1 / 3}\left(s-s_{0}\right)\right)} .
$$

We complete our hydrodynamic description of the Dyson fluid by considering the Cauchy transforms of the monic orthogonal polynomials

$$
p_{k}(z, \tau)=\frac{1}{2 \pi i} \int_{-\infty}^{\infty} d x \frac{\pi_{k}(x, \tau) e^{-N x^{2} / 2 \tau}}{x-z} .
$$

The motivation for doing so is that the average of the inverse characteristic polynomial is related to $p_{N-1}$ [16]:

$$
\left\langle\frac{1}{\operatorname{det}(z-H(\tau))}\right\rangle=-\frac{2 \pi i}{c_{N-1}^{2}} p_{N-1}(z, \tau),
$$

where $c_{N-1}^{2}$ is given after Eq. (17). To get the time dependent version of the $p_{k}$ 's, we plug into Eq. (28) the integral representation of the time-dependent monic polynomials $\pi_{x}(x, \tau)$. A simple calculation then yields, for $\operatorname{Im} z>0$,

$$
p_{k}(z)=(-i)^{k} \sqrt{\frac{N}{2 \pi \tau}} \int_{0}^{\infty} d q q^{k} e^{-\frac{N}{2} \tau\left(q^{2}-2 i q z\right)},
$$

and $p_{k}(-z, \tau)=(-1)^{k+1} p_{k}(z, \tau)$. It can be shown by a direct calculation that the functions

$$
\tilde{p}_{k}(z, \tau)=e^{\frac{N}{2 \tau} z^{2}} p_{k}(z, \tau)
$$

satisfy the same recurrence relations and differential equations as the polynomials $\pi_{k}(z, \tau)$, in particular Eq. (18). One may also verify that the function

$$
g_{k}(z, \tau)=2 v_{s} \partial_{z} \ln \tilde{p}_{k}(z, \tau),
$$


analogous to $f_{k}(z, \tau)$ in Eq. (19), satisfies a viscid Burgers equation:

$$
\partial_{\tau} g_{k}+g_{k} \partial_{z} g_{k}=-v_{s} \partial_{z}^{2} g_{k}
$$

Also this equation exhibits the phenomenon of universal preshock, but this time the scaling solution involves the two other solutions of the Airy equation that were mentioned earlier, namely $\phi_{1}$ or $\phi_{2}$, depending on the sign of the imaginary part of $z$, in agreement with [15].

We note that since $\pi_{N}(z)$ and $p_{N}(z)$, and their first derivatives, are the building blocks of all relevant multipoint correlators (products of characteristic polynomials, products of inverse characteristic polynomials and mixed products [16]), all universal kernels originate implicitly from the dynamics of shocks in the viscid Dyson fluid. Also, the so-called "hardedge" scaling (Bessel kernels) can be be viewed in the same way, as a pre-shock approaching a hard wall. We note also that other forms of universal shocks may appear, e.g., when the spectral density develops a cubic root singularity; then the universal character of the phenomenon is coded in a Pearcey function. This happens e.g. when two intervals supporting the hermitian spectrum merge as a function of an external parameter [11, 12], or when two Airy type shock waves merge on a compact support [9, 13].

We expect the fluid dynamical picture presented here to hold also for other matrix-valued diffusion processes, in particular for the multiplicative diffusions, corresponding to random walks involving products of complex matrices [17, 18]. Perhaps the best studied example is the random walk of unitary matrices [19], because of its relation with two dimensional Yang-Mills theory with a large number of col- ors $N_{c}$ [20], and the associated universality conjectured by Narayanan and Neuberger [13]. We have earlier emphasized the relevance of shock waves in this context [9], as providing a simple mechanism for the Durhuus-Olesen [21] orderdisorder transition. Recently, Neuberger used the explicit expressions of averages of the characteristic polynomial and its inverse, that were obtained through a character expansion, to prove that closely related functions satisfy Burgers equations with a spectral viscosity $1 / 2 N_{c}$ [22, 23]. The present analysis supports the general character of these results and may motivate further generalizations. In particular, we expect that preshock phenomena may be identified for non-compact random matrix models, e.g. in mesoscopic systems where the role of the time is played by the length of the wire [24]. Last but not least, this picture of shock formation finds analogies in other branches of physics, e.g., in the descritption of merging singularities in optics, leading to the so-called diffraction catastrophes (see e.g. [25]), or in the study of growth processes of the Kardar-Parisi-Zhang universality class and statistical properties of the equilibrium shapes of crystals [26]. We believe that pushing Dyson's original concept of temporal dynamics for random matrices may allow for a better understanding of above analogies.

\section{Acknowledgements}

We would like to thank Romuald A. Janik and Roland Speicher for illuminating remarks. MAN thanks IPhT for hospitality during the time this paper was completed. This work was supported by Marie Curie TOK Grant MTKD-CT-2004517186 “Correlations in Complex Systems” (COCOS).
[1] F.J. Dyson, J. Math. Phys. 3 (1962) 1191.

[2] For a review, see T. Guhr, A. Mueller-Groeling and H.A. Weidenmueller, Phys. Rept. 299 (1998) 189.

[3] D. Bessis and J.D. Fournier, J. Physique Lett. 45 (1984) L833.

[4] Y. V. Fyodorov, e-print math-ph/0412017

[5] J.M. Burgers, The Nonlinear diffusion equation, D. Reidel Publishing Company (1974).

[6] D. V. Voiculescu, K. J. Dykema and A. Nica, Free Random Variables, CRM Monograph Series, Vol.1, Am. Math. Soc., Providence, 1992.

[7] P. Biane and R. Speicher, Ann. Inst. H. Poincaré PR 37 (2001) 581.

[8] E. Brezin and S. Hikami, Commun. Math. Phys. 214 (2000) 111.

[9] J.-P. Blaizot and M.A. Nowak, Phys. Rev. Lett. 101 (2008)100102.

[10] S.J. Chapman, C.J. Howls, J.R. King and A.B. Olde Daalhuis, Nonlinearity, 20 (2007) 2425.

[11] E. Brezin and S. Hikami, Phys. Rev. E57 (1998) 4140.

[12] R.A. Janik, M.A. Nowak, G. Papp and I. Zahed, Phys. Lett. B446 (1999)9.

[13] R. Narayanan and H. Neuberger, JHEP 0712 (2007) 066.
[14] Y.V. Fyodorov and E. Strahov, J. Phys. E36 (2003) 3203.

[15] G. Akemann and Y.V. Fyodorov, Nucl. Phys.B664 (2003) 457.

[16] Y.V. Fyodorov and E. Strahov, Comm. Math. Phys. 241 (2003).

[17] E. Gudowska-Nowak, R.A. Janik, J. Jurkiewicz and M.A. Nowak, Nucl. Phys. B670 (2003) 479; New Jour. of Phys., 7 (2005) 54.

[18] R. Lohmayer, H. Neuberger and T. Wettig, JHEP 0811:053 (2008)

[19] R. A. Janik and W. Wieczorek, J. Phys. A: Math. Gen. 37 (2004) 6521.

[20] R. Gopakumar and D. Gross, Nucl. Phys. B 451 (1995) 379 and references therein.

[21] B. Durhuus and P. Olesen, Nucl. Phys. B184 (1981) 461.

[22] H. Neuberger, Phys. Lett. B666 (2008) 106.

[23] H. Neuberger, Phys. Lett. B670 (2008) 235.

[24] C.W.J. Beenakker, Rev. Mod. Phys. 69 (1997) 731.

[25] see e.g. M.V. Berry and S. Klein, Proc. Natl. Acad. Sci. USA 93 (1996) 261.

[26] H. Spohn, e-print cond-mat/0512011

[27] O. Vallee and M. Soares, Airy functions and applications to physics, Imperial College Press, 2004. 